\title{
Mécanismes d'action des leucotriènes
}

Les leucotriènes sont des dérivés de l'acide arachidonique jouant un rôle de médiateurs dans la réaction inflammatoire, et probablement dans de nombreux autres phénomènes cellulaires. Ils se fixent à des récepteurs spécifiques de haute affinité couplés, pour la plupart, à l'activation du cycle des phospho-inositides. L'un des leucotriènes, LTC $_{4}$, ne semble cependant pas agir selon ce schéma ; ses récepteurs, présents sur les membranes plasmiques et sur celles d'organites intracellulaires, n'apparaissent pas couplés de la même manière et le mécanisme d'action de ce médiateur reste très mystérieux. Certains effets des leucotriènes sont liés à l'activation de la phospholipase $A_{2}$ et à la libération extracellulaire de prostanoïdes tel le thromboxane $\mathbf{A}_{2}$.

\section{Agnès Muller Claude Bonne}

A. Muller : Docteur ès sciences, ingénieur de recherche. C. Bonne: Professeur. Groupement scientifique CNRS leucotriènes, laboratoire de physiologie cellulaire, université Montpellier I, 15, avenue Charles-Flahault, 34060

es leucotriènes, dérivés de l'acide arachidonique, sont d'importants médiateurs de l'inflammation et des modulateurs ubiquiaires des fonctions cellulaires. Ils se lient à des protéines membranaires spécifiques et induisent la plupart de leurs effets par activation du cycle des phospho-inositides ainsi que par la stimulation de la synthèse des prostanoïdes. La connaissance de leurs mécanismes d'action, et particulièrement de leurs récepteurs, a permis de concevoir des antagonistes spécifiques qui pourraient avoir un intérêt thérapeutique notamment dans l'asthme.

Les leucotriènes (LT) sont un groupe de métabolites de l'acide arachidonique formés par la voie de la 5-lipoxygénase (figure 1). On distingue deux types de leucotriènes, selon qu'ils comportent une chaîne peptidique $\left(\mathrm{LTC}_{4}, \mathrm{LTD}_{4}, \mathrm{LTE}_{4}\right)$ ou non $\left(\mathrm{LTB}_{4}\right)$. Tous dérivent d'un précurseur commun, époxyde instable, le $\mathrm{LTA}_{4}$. Ces dérivés, dont la structure n'a été élucidée qu'en 1979 par le groupe de Samuelsson, sont les constituants de la slow reacting substance of anaphylaxis (SRS-A), agenlt contracturant des muscles lisses connu depuis un demi-siècle. Si les leucotriènes parti- cipent en effet en tant que médiateurs de l'inflammation à un grand nombre de phénomènes pathologiques dont l'asthme, ils semblent impliqués également dans la régulation physiologique de multiples fonctions cellulaires [1].

Cependant, leurs mécanismes d'action ont été essentiellement étudiés dans les cellules qui participent aux réactions inflammatoires et anaphylactiques. Ainsi, le mécanisme d'action du $\mathrm{LTB}_{4}$, médiateur qui intervient dans l'inflammation en tant qu'activateur leucocytaire, a surtout été décrit dans les polynucléaires neutrophiles, alors que celui des peptidoleucotriènes, a été principalement étudié sur les muscles lisses des voies aériennes.

\section{Sites de liaison des leucotriènes}

Bien que certains effets biologiques induits par les leucotriènes à des concentrations inférieures à $10^{-12} \mathrm{M}$, n'aient pas pu encore être reliés, pour des raisons techniques, à la présence de sites de liaison (action neurosécrétrice du $\mathrm{LTC}_{4}$ [2], effet prolifératif du $\mathrm{LTB}_{4}$ sur les lymphocytes T [3]), de tels sites de liaison spécifiques ont été caractérisés pour les différents leuco- 


\begin{tabular}{|c|c|c|c|c|c|c|}
\hline \multicolumn{7}{|c|}{$\begin{array}{c}\text { Tableau I } \\
\text { SITES DE LIAISON DES LEUCOTRIENES }\end{array}$} \\
\hline $\begin{array}{l}\text { Tissu et } \\
\text { cellules }\end{array}$ & Espèce & Ligand & $\mathbf{K d}(\mathrm{nM})$ & $\begin{array}{c}\text { Bmax } \\
\text { (pmol/mg prot) } \\
\text { (" sites/cellule) }\end{array}$ & Spécificité & Référence \\
\hline Bronches & $\mathrm{H}$ & $\mathrm{LTC}_{4}$ & 70 & 184 & $C_{4}>D_{4}>E_{4}$ & $\begin{array}{l}\text { Civelli et al. J Pharmacol Exp Ther } 1987 ; 242 \text { : } \\
1019 .\end{array}$ \\
\hline Poumon & $\begin{array}{l}\mathrm{R} \\
\mathrm{C}\end{array}$ & $\begin{array}{l}\mathrm{LTC}_{4} \\
\mathrm{LTC}_{4} \\
\mathrm{LTD}_{4} \\
\mathrm{LTB}_{4} \\
\mathrm{LTC}_{4} \\
\mathrm{LTO}_{4}\end{array}$ & $\begin{array}{c}41 \\
15 \\
\\
52 \\
0,2 \\
1,8 \\
0,28 \\
0,76 \\
26 \\
36 \\
0,1\end{array}$ & $\begin{array}{c}31 \\
68 \\
30 \\
0,3 \\
2,1 \\
0,06 \\
- \\
84 \\
32 \\
0,06\end{array}$ & $\begin{array}{l}C_{4}>D_{4} \\
C_{1}>C_{4}>D_{4}=E_{4} \\
C_{4}>D_{4}>E_{4}>G S H \\
D_{4}>E_{4} \\
D_{4}>E_{4} \\
D_{4}=C_{4}>E_{4} \\
8 B_{4}>20 O H B_{4}>20 \\
\text { COOH } B_{4} \\
2 \mathrm{Nor} \mathrm{C}_{1}>C_{4}>D_{4} \\
C_{4}>D_{4}>E_{4} \\
D_{4}>E_{4}\end{array}$ & $\begin{array}{l}\text { Pong et al. J Biol Chem } 1983 ; 258: 9616 . \\
\text { Mong et al. J Pharmacol Exp Ther } 1985 ; 234 \text { : } \\
316 . \\
\text { Norman et al. Eur J Pharmacol } 1987 ; 143: 323 . \\
\text { Pong et al. Proc Natl Acad Sci USA } 1983 ; 80 \text { : } \\
7415 \text {. } \\
\text { Mong et al. Eur J Pharmacol } 1984 ; 102: 1 \text {. } \\
\text { Cheng et al. Biochem Biophys Res Commun } \\
1984 ; 118: 20 \text {. } \\
\text { Saad et al. Biochem Biophys Res Commun 1987; } \\
143: 364 \text {. } \\
\text { Lewis et al. Prostaglandins } 1984 ; 27: 961 . \\
\text { Rovati et al. Biochem Pharmacol } 1985 ; 34: \\
2831 . \\
\text { Lewis et al. Biochem Pharmacol } 1985 ; 34: \\
4311 .\end{array}$ \\
\hline Iléon & $\mathrm{C}$ & $\mathrm{LTC}_{4}$ & 1,3 à 13 & 0,1 à 1,6 & $\mathrm{C}_{4}>\mathrm{D}_{4}>\mathrm{GSH}>\mathrm{E}_{4}$ & $\begin{array}{l}\text { Krilis et al. Proc Natl Acad Sci USA 1984; } 81 \text { : } \\
4529 .\end{array}$ \\
\hline Utérus & C & $\mathrm{LTC}_{4}$ & 5 & - & $\mathrm{C}_{4}>\mathrm{D}_{4}$ & Levinson Pharmacologist 1983 ; 25 : 201. \\
\hline $\begin{array}{l}\text { Cellules musculaires } \\
\text { lisses (culture) }\end{array}$ & $\begin{array}{l}\mathrm{DDT}_{1} \mathrm{MF}_{2} \\
\mathrm{BC}_{3} \mathrm{H}_{1}\end{array}$ & $\begin{array}{l}\mathrm{LTC}_{4} \\
\mathrm{LTC}_{4} \\
\mathrm{LTD}_{4}\end{array}$ & $\begin{array}{c}5 \\
21 \\
33 \\
14,3 \\
9,3\end{array}$ & $\begin{array}{c}7000 \\
55 \\
25 \\
4 \\
0,37\end{array}$ & $\begin{array}{l}C_{4}>D_{4}>E_{4} \\
C_{4} \gg E_{4}>D_{4} \\
C_{4} \gg E_{4}>D_{4} \\
C_{4} \gg D_{4}>E_{4} \\
D_{4}=E_{4}>>C_{4}\end{array}$ & $\begin{array}{l}\text { Krilis et al. J Clin Invest } 1983 ; 72: 1516 . \\
\text { Clark et al. Life Sci } 1984 ; 35: 441 . \\
\text { Clark et al. Eur J Pharmacol 1985; } 116: 207 . \\
\text { Tamura et al. Life Sci } 1987 ; 41: 207 . \\
\text { Tamura et al. Life Sci } 1987 ; 41: 207 .\end{array}$ \\
\hline Cour & C & $\begin{array}{l}\mathrm{LTC}_{4} \\
\mathrm{LTD}_{4}\end{array}$ & $\begin{array}{c}27,5 \\
3,4\end{array}$ & $\begin{array}{c}20 \\
0,85\end{array}$ & $\begin{array}{l}C_{4}>D_{4}=E_{4} \\
D_{4}>E_{4}>C_{4}\end{array}$ & $\begin{array}{l}\text { Hogaboom et al. Mol Pharmacol } 1985 ; 27: 236 . \\
\text { Hogaboom et al. J Pharmacol Exp Ther } 1985: \\
233: 686 .\end{array}$ \\
\hline $\begin{array}{l}\text { Rein } \\
\text { Cellules épithé. } \\
\text { liales }\end{array}$ & $\begin{array}{l}R \\
H\end{array}$ & $\begin{array}{l}\operatorname{LTC}_{4} \\
\operatorname{LTC}_{4}\end{array}$ & $\begin{array}{c}25 \\
220\end{array}$ & $\begin{array}{l}8,5 \\
4,5\end{array}$ & $\begin{array}{l}C_{4}>D_{4}=E_{4} \\
C_{4}>>E_{4}>D_{4}\end{array}$ & $\begin{array}{l}\text { Ballerman et al. Circ Res } 1985 ; 56: 324 . \\
\text { Baud et al. J Clin Invest } 1985 ; 76: 374 .\end{array}$ \\
\hline Rate & $\mathrm{C}$ & $\mathrm{LTB}_{4}$ & 1,8 & 0,3 & $\mathrm{~B}_{4}>20 \mathrm{OH} \mathrm{B}{ }_{4}>\mathrm{A}_{4}$ & $\begin{array}{l}\text { Cheng et al. J Pharmacol Exp Ther } 1986 ; 236 \text { : } \\
126 .\end{array}$ \\
\hline Foie & $\mathrm{R}$ & $\mathrm{LTC}_{4}$ & 0,77 & 145 & $\mathrm{C}_{4} \gg \mathrm{D}_{4}>\mathrm{E}_{4}$ & Sun et al. J Biol Chem 1986 ; $261: 8540$. \\
\hline Cerveau & $\begin{array}{l}S \\
R\end{array}$ & $\begin{array}{l}\mathrm{LTC}_{4} \\
\mathrm{LTC}_{4}\end{array}$ & $\begin{array}{l}10,4 \\
31,4\end{array}$ & $\begin{array}{l}49,2 \\
0,041\end{array}$ & $\begin{array}{l}\mathrm{C}_{4}>\mathrm{D}_{4}>\mathrm{GSH} \\
\mathrm{C}_{4}>\mathrm{D}_{4}=\mathrm{E}_{4}\end{array}$ & $\begin{array}{l}\text { Goffinet et al. Eur J Pharmacol } 1987 ; 140: 343 . \\
\text { Schalling et al. Eur J Pharmacol 1986; } 122 \text { : } \\
251\end{array}$ \\
\hline $\begin{array}{l}\text { Cellules } \\
\text { endothéliales } \\
\text { vasculaires (culture) }\end{array}$ & B & $\mathrm{LTC}_{4}$ & $\begin{array}{c}6,8 \\
49,9\end{array}$ & $\begin{array}{l}* 2000 \\
24,5\end{array}$ & $\begin{array}{l}C_{4}>D_{4}>E_{4} \\
C_{4}>G^{\prime}>D_{4}\end{array}$ & $\begin{array}{l}\text { Chau et al. J Immunol } 1986: 137: 1985 . \\
\text { Muller et al. Prostagland Leuk Med } 1987 ; 26 \text { : } \\
233 .\end{array}$ \\
\hline $\begin{array}{l}\text { Kératinocytes } \\
\text { (culture) }\end{array}$ & $\mathrm{H}$ & $\mathrm{LTC}_{4}$ & 8.7 & 1,2 & $C_{4}>D_{4}>B_{4}>E_{4}$ & Muller et al. Br J Dermatol 1988 ; 119: 275. \\
\hline Macrophages & $\mathrm{H}$ & $\mathrm{LTD}_{4}$ & 3,8 & 1,2 & $\mathrm{D}_{4}>\mathrm{C}_{4}$ & Opmeer et al. Prostaglandins $1984 ; 28$ : 183. \\
\hline \multirow[t]{2}{*}{$\begin{array}{l}\text { Polynucléaires } \\
\text { Neutrophiles }\end{array}$} & $\begin{array}{l}R \\
H\end{array}$ & $\begin{array}{l}\mathrm{LTB}_{4} \\
\mathrm{LTB}_{4} \\
\mathrm{LTC}_{4}\end{array}$ & $\begin{array}{l}4,5 \\
0,46 \\
0,39 \\
34\end{array}$ & $\begin{array}{l}* 8200 \\
* 20000 \\
* 3600 \\
* 10000\end{array}$ & $\begin{array}{l}\mathrm{B}_{4}>20 \mathrm{OH} \mathrm{B} \mathrm{B}_{4} \\
\mathrm{~B}_{4}>20 \mathrm{OH} \mathrm{B} \mathrm{B}_{4} \\
\mathrm{~B}_{4}>12(\mathrm{~S}) \mathrm{B}_{4}>5 \\
\mathrm{HETE} \\
\mathrm{C}_{4}>\mathrm{D}_{4}>\mathrm{E}_{4}\end{array}$ & $\begin{array}{l}\text { Kreisle et al. J Immunol } 1985 ; 184: 3356 . \\
\text { Lin et al. Prostaglandins } 1984 ; 28: 837 . \\
\text { Goldman et al. J Exp Med } 1984 ; 159: 1027 . \\
\text { Baud et al. Immunology } 1987 ; 62: 53 .\end{array}$ \\
\hline & $\begin{array}{l}\mathrm{HL}_{60} \\
\mathrm{RBL}_{1}\end{array}$ & $\begin{array}{l}\mathrm{LTB}_{4} \\
\mathrm{LTD}_{4}\end{array}$ & $\begin{array}{c}0,27 \\
0,9\end{array}$ & $\begin{array}{c}24 \\
0,8\end{array}$ & $\begin{array}{l}\mathrm{B}_{4}>20 \mathrm{OH} \mathrm{B} \mathrm{B}_{4} \\
\mathrm{D}_{4}>\mathrm{C}_{4}>\mathrm{E}_{4}\end{array}$ & $\begin{array}{l}\text { Benjamin et al. J Biol Chem 1985; } 260: 14208 . \\
\text { Sarau et al.J Biol Chem 1987; } 262: 4034 .\end{array}$ \\
\hline Hématies & $\mathrm{H}$ & $\mathrm{LTC}_{4}$ & 15,9 & 0,97 & $\begin{array}{l}\mathrm{C}_{4}>\mathrm{GSH}>\mathrm{GS} \cdot \mathrm{SG} \\
>>\mathrm{D}_{4}\end{array}$ & $\begin{array}{l}\text { Ghiglieri-Bertez et al. Biochim Biophys Acta } \\
1986 ; 879: 97 \text {. }\end{array}$ \\
\hline
\end{tabular}

$H=$ homme $R=$ rat,$S=$ souris, $C=$ cobaye, $B=$ bœuf.

$\mathrm{m} / \mathrm{s} n^{\circ} 1$ vol. 5 , janvier 89 


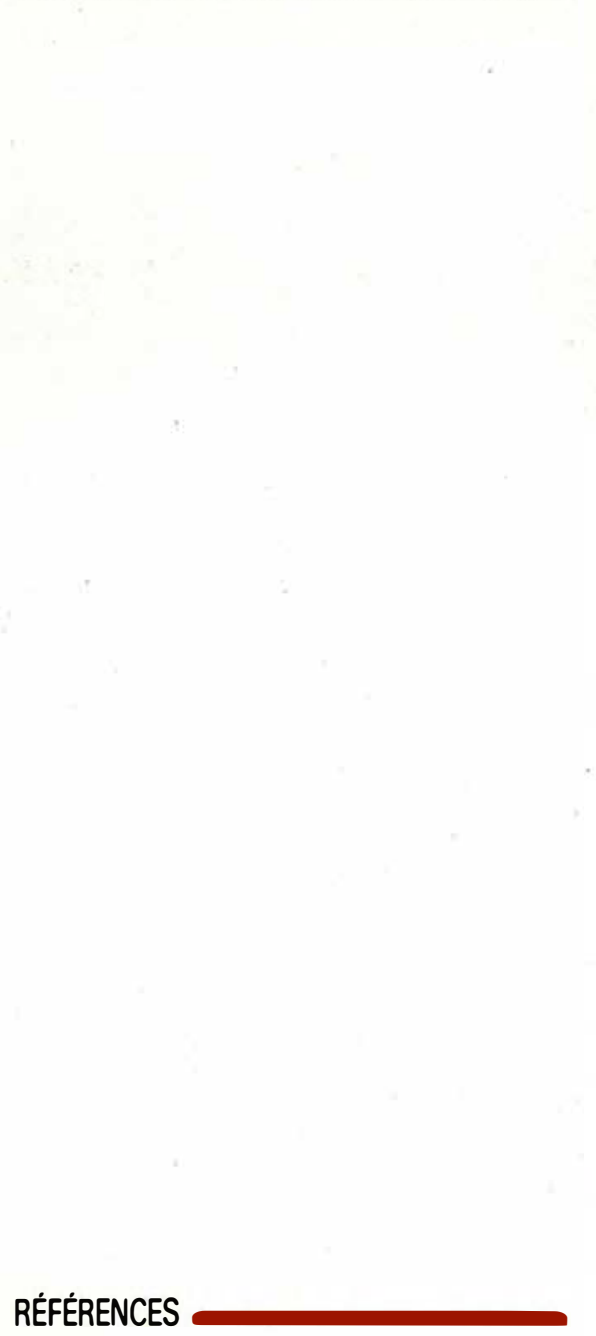

1. Samuelsson B, Dahlen SE, Lindgren JA, Rouzer CA, Serhan CN. Leukotrienes and lipoxins : structures, biosynthesis, and biological effects. Science 1987 ; 237 : 1171-6.

2. Gerozissis K, Saadi M, Dray F. Leukotrienes $\mathrm{C}_{4}$ and $\mathrm{D}_{4}$ stimulate the release of luteinizing hormone-releasing hormone from rat median eminence in vitro. Brain Res 1987 ; 416 : $54-8$.

3. Payan DG, Missirian-Bastian A, Goetzl EJ. Human T-lymphocyte subset specificity of the regulatory effects of leukotriene B. Proc Natl Acad Sci USA 1984; 81 : 3501.

4. Bomalaski JS, Mong S. Binding of leukotriene $\mathrm{B}_{4}$ and its analogs to human polymorphonuclear leukocyte membrane receptors. Prostaglandins 1987; 33 : 855-67.

5. Mong S, Wu HL, Clark MA, Gleason JG, Crooke ST. Leukotriene $\mathrm{D}_{4}$ receptor-mediated synthesis and release of arachidonic acid metabolites in guinea-pig lung : induction of thromboxane and prostacyclin biosynthesis by leukotriene $\mathrm{D}_{4}$. J Pharmacol Exp Ther

\section{ACIDE ARACHIDONIQUE}
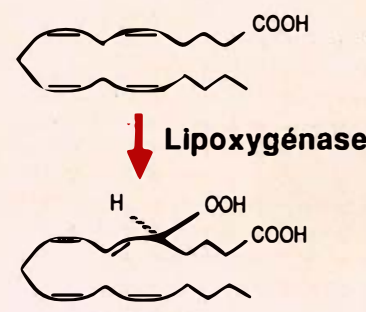

5. HPTE

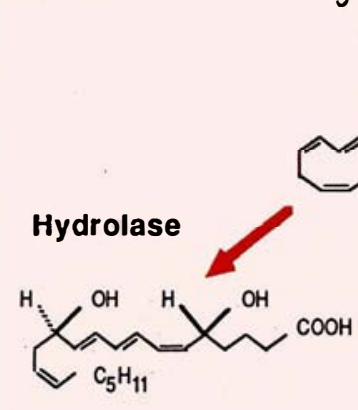

Déhydrase

象

LTA4

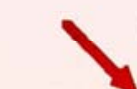

Glutathion

S-transférase

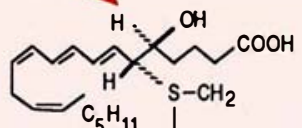

$\mathrm{CHCONHCH}_{2} \mathrm{COOH}$

$\mathrm{NHCOCH}_{2} \mathrm{CH}_{2} \mathrm{CHCOOH}$

LTC4

$\mathrm{NH}_{2}$

GGTP
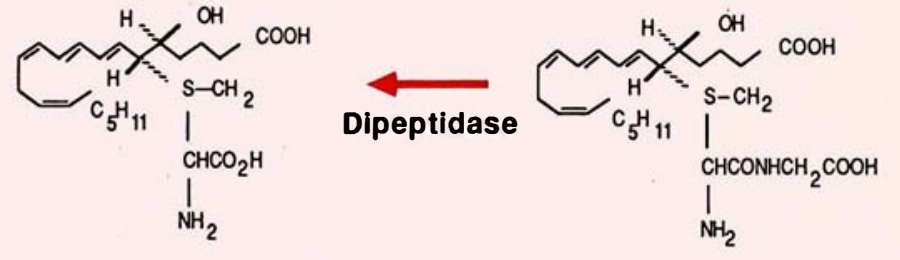

LTD4

Figure 1. Biosynthèse des leucotriènes.

triènes dans les membranes de la plupart de leurs tissus cibles (Tableau I)

Ces sites présentent une affinité élevée pour leurs ligands respectifs et une grande spécifícité pour chacun d'eux. Le $\mathrm{LTE}_{4}$ est toutefois reconnu par les mêmes sités que le $\mathrm{LTD}_{4}$. Une relation directe a été établie entre l'affinité d'analogues du $\mathrm{LTB}_{4}$ et du $\mathrm{LTD}_{4}$ pour leurs sites de liaison respectifs et leurs activités biologiques [4, 5]. De même, des antagonistes de synthèse inhibent parallèlement la liaison des ligands et les effets qu'ils induisent [6]. Ces données concourent à considérer les sites de liaison du $\mathrm{LTB}_{4}$ et du $\mathrm{LTD}_{4} / \mathrm{E}_{4}$ comme des sites récepteurs.

En revanche, le rôle potentiel des sites de liaison du $\mathrm{LTC}_{4}$ est moins aisément interprétable. Ces sites ne sont pas seulement localisés sur les membranes plasmiques mais aussi sur les membranes intracellulaires et sur les mitochondries [7-9]. Contrairement aux liaisons des leucotriènes précédents, celles du $\mathrm{LTC}_{4}$ sont insensibles à la présence de nucléotides guanyliques. Une diminution 


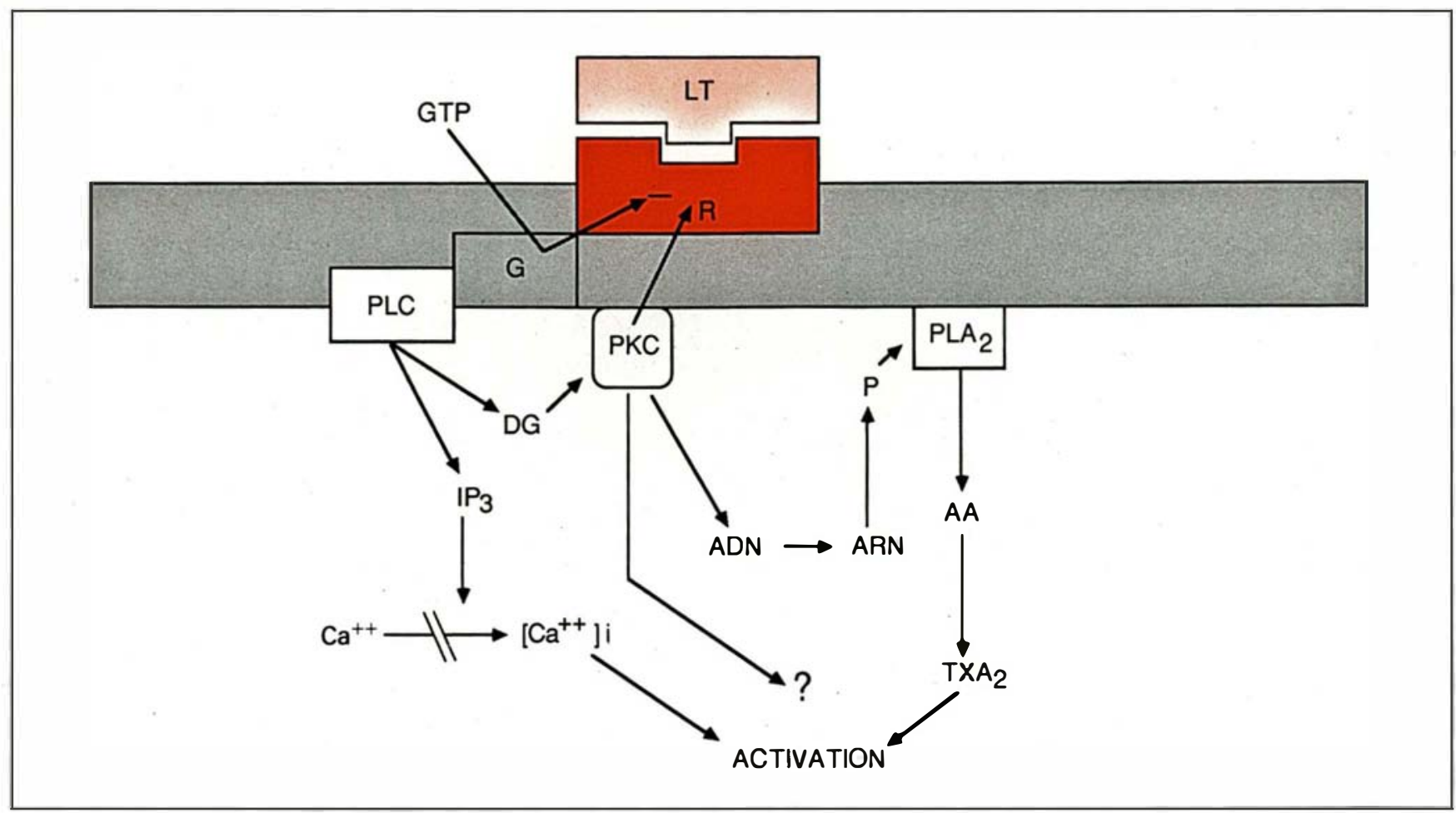

Figure 2. Mécanismes d'action des leucotriènes. LTB $_{4}$. Les récepteurs membranaires du $L_{T} B_{4}(R)$ sont couplés à une protéine régulatrice sensible au GTP (G), permettant la transduction du signal par formation de messagers intracellulaires. Dans la plupart des cas étudiés, la G-protéine active la phospholipase C (PLC) et le cycle des phospho-inositides avec libération d'inositol triphosphate $\left(I P_{3}\right)$ et de diacylglycérol $(D G)$ qui induisent respectivement une mobilisation du calcium et une activation de la protéine kinase $C$ (PKC). L'activation de la PKC entraîne notamment une régulation négative du système de reconnaissance membranaire. Le calcium libéré [C $\left.\mathrm{Ca}^{++}\right]$i peut conduire à la réponse cellulaire par activation de diverses kinases et phospholipases, en particulier de la phospholipase $A_{2}\left(P L A_{2}\right)$. La libération d'acide arachidonique (AA) qui en découle permet la synthèse du thromboxane $A_{2}\left(T X A_{2}\right)$ responsable des effets contracturants du LTB L LTD $_{4} / E_{4}$. Les leucotriènes LTD ${ }_{4}$ et $L_{T E}$ agissent par fixation à des sites spécifiques membranaires couplés par une G-protéine à la PLC. Cette interaction active le cycle des phospho-inositides avec formation d'IP ${ }_{3}$, qui mobilise le calcium intracellulaire, et de DG, activateur de la PKC. Calcium et PKC contribuent à la stimulation de la synthèse d'une protéine (P) activant la PLA $A_{2}$ et la formation de prostanoïdes qui participent à certaines actions de ces LT.

d'affinité en présence de guanosine triphosphate (GTP) est un élément en faveur d'un couplage, par une Gprotéine, entre un récepteur membranaire et une enzyme productrice de seconds messagers intracellulaires. Le caractère fonctionnel des sites de liaison du $\mathrm{LTC}_{4}$ est encore rendu plus ambigu par leur très large répartition tissulaire et le fait que la glutathion S-transférase cytosolique et membranaire lie également ce leucotriène avec une haute affinité [10]. Des mesures analytiques suggèrent que certains effets du $\mathrm{LTC}_{4}$ sont probablement induits par l'intermédiaire du $\mathrm{LTD}_{4}$, car sa transformation métabolique par la $\gamma$-glutamyltranspeptidase $(\gamma \mathrm{GTP})$ est rapide et ubiquitaire. Cependant le $\mathrm{LTC}_{4} \mathrm{sem}$ ble capable d'agir par ses propres sites de liaison, puisqu'il conserve ses $\mathrm{m} / \mathrm{s} n^{\circ} 1$ vol. 5 , janvier 89 effets en présence d'inhibiteurs de $\gamma \mathrm{GTP}$, et que, dans certains modèles, le $\mathrm{LTC}_{4}$ est plus puissant que ses métabolites [11].

\section{Transduction par activation du cycle des phospho-inositides}

Les signaux déclenchés par la liaison membranaire du $\mathrm{LTB}_{4}$ et du $\mathrm{LTD}_{4}$ semblent être le plus souvent transmis par l'activation d'une phosphodiestérase spécifique $\left(\mathrm{PIP}_{2}-\mathrm{PDE}\right.$ ou PLC) qui stimule le cycle des phospho-inositides et la synthèse de seconds messagers intracellulaires tels que l'inositol 1, 4, 5-triphosphate $\left(\mathrm{IP}_{3}\right)$ et le sn-l, 2-diacylglycérol (DG) (figure 2).

$\mathbf{L T B}_{4}$. Il a d'abord été démontré que le $\mathrm{LTB}_{4}$ augmente rapidement la concentration du calcium $\left[\mathrm{Ca}^{++}\right]_{i}$ dans les polynucléaires neutrophiles par mobilisation des pools du réticulum endoplasmique. Cet effet est stéréospécifique et peut être inhibé par la toxine de Bordetella pertussis (IAP) [12], qui abolit la fonction de G-protéines assurant en particulier le couplage de récepteurs à la PLC. Ces observations, qui suggèrent que la mobilisation calcique induite par le $\mathrm{LTB}_{4}$ est la conséquence de l'activation du cycle des phospho-inositides, ont été confirmées dans d'autres expériences: le $\mathrm{LTB}_{4}$ et ses analogues induisent la formation $\mathrm{d}^{\prime} \mathrm{IP}_{3}$, synthèse qui est effectivement inhibée par le IAP et précède la mobilisation du calcium [13].

Le DG, autre second messager engendré par l'activation du cycle 
6. Perchonock CD, Torphy T J, Mong S. Peptidoleukotrienes : pathophysiology, receptor biology and receptor antagonists. Drugs Future $1987 ; 12$ : 871-89.

7. Krilis S, Lewis RA, Corey EJ, Austen KF. Specific binding of leukotriene $\mathrm{C}_{4}$ to ileal segments and subcellular fractions of ileal smooth muscle cells. Proc Natl Acad Sci USA 1984; 81 : 4529-33.

8. Chau LY, Hoover RL, Austen KF, Lewis RA. Subcellular distribution of leukotriene $\mathrm{C}_{4}$ binding units in cultured bovine aortic endothelial cells. J Immunol $1986 ; 137$ : 1985 92.

9. Baud L, Koo CH, Goetzl EJ. Specificity and cellular distribution of human polymorphonuclear leucocyte receptors for leukotriene $\mathrm{C}_{4}$. Immunology 1987; 62 : 53-9.

10. Sun FF, Chau LY, Austen KF. Binding of leukotriene $\mathrm{C}_{4}$ by glutathione transferase : a reassessment of biochemical and functional criteria for leukotriene receptors. Fed Proc. $1987 ; 46$ : 204-7.

11. Ballermann BJ, Lee TH, Lewis RA, Austen KF. Distinct sulfidopeptide leukotriene receptors. In : Bayley JM, ed. Prostaglandins. Leukotrienes and lipoxins. New York: Raven Press, 1985 : $311-20$.

12. Goldman DW, Chang FH, Gifford LA, Goetzl EJ, Bourne HR. Pertussis toxin inhibition of chemotactic factor-induced calcium mobilization and function in human poly morphonuclear leukocytes. J Exp Med 1985 ; $162: 145-56$

13. Andersson T, Schlegel W, Monod A, Krause KH, Stendhal O, Lew DP. Leukotriene $\mathrm{B}_{4}$ stimulation of phagocytes results in the formation of inositol 1, 4,5-triphosphate. A second messenger for $\mathrm{Ca}^{2+}$ mobilization. Biochem J 1986 ; 240 : 333-40.

14. Holian A. Leukotriene $B_{4}$ stimulation of phosphatidylinositol turnover in macrophages and inhibition by pertussis toxin. FEBS Lett 1986; 201 : 15-9.

15. O'Flaherty JT, Redman JF, Jacobson DP. Protein kinase $C$ regulates leukotriene $B_{4}$ receptors in human neutrophils. FEBS Lett 1986 ; 206 : 279-82.

16. Sarau HM, Mong S, Foley JJ, Wu HL, Crooke ST. Identification and characterization of leukotriene $\mathrm{D}_{4}$ receptors and signal transduction processes in rat basophilic leu-

17. Mong S, Hoffman K, Wu HL, Crooke ST. Leukotriene-induced hydrolysis of inositol lipids in guinea-pig lung: mechanism of signal transduction for leukotriene $\mathrm{D}_{4}$ receptors. Mol Pharmacol 1987 ; 31 : 35-4l.

18. Mong S, Miller J, Wu HL, Crooke ST. Leukotriene $\mathrm{D}_{4}$ receptor-mediated hydrolysis of phosphoinositide and mobilization of calcium in sheep tracheal smooth muscle cells. $J$ Pharmacol Exp Ther 1988; $244: 508-15$.

19. Sirois $\mathrm{P}$, Chagnon $\mathrm{M}$, Borgeat $\mathrm{P}$, Vallerand $\mathrm{P}$. Role of cyclooxygenase products in the lung action of leukotrienes $A_{4}, B_{4}, C_{4}, D_{4}$ and $\mathrm{E}_{4}$. Pharmacology 1985; 31 : 225-36.

20. Modat G, Muller A, Mary A, Bonne C. $\mathrm{LTC}_{4}$, but not $\mathrm{LTB}_{4}$, binds vascular endothelial cells and promotes their proliferation in vitro. Ann NY Acad Sci 1988 ; 524 : 414-6.

21. Clark MA, Cook M, Mong S, Crooke ST. The binding of leukotriene $\mathrm{C}_{4}$ and leukotriene $\mathrm{D}_{4}$ to membranes of a smooth muscle cell line $\left(\mathrm{BC}_{3} \mathrm{H}_{1}\right)$ and evidence that leukotriene-induced contraction in these cells is mediated by thromboxane, protein and RNA synthesis. Eur J Pharmacol 1985; 116: 207-20.

22. Clark MA, Conway TM, Shorr RG, Crooke ST. Identification and isolation of a mammalian protein which is antigenically and functionally related to the phospholipase $\mathrm{A}_{2}$ stimulatory peptide melitin. J Biol Chem $1987 ; 262$ : 4402-6.

23. Claesson HE, Feinmark SJ. Relationship of cyclic-AMP levels in leukotriene $B_{4}$-stimulated leukocytes to lysosomal enzyme release and the generation of superoxide anions. Biochim Biophys Acta 1984; 804 : 52-7.

24. Serhan CN, Fridovich J, Goetzl EJ, Dunham PB, Weissmann $G$. Leukotriene $B_{4}$ and phosphatidic acid are calcium ionophores. Studies employing arsenazo III in liposomes. J Biol Chem 1982 ; 257 : 4746-52.

25. Holmes RP, Yoss NL, Marshall LA. Failure of leukotriene $\mathrm{B}_{4}$ to translocate calcium across phosphatidylcholine membranes. Cell Calcium $1987 ; 8$ : 449-54

26. Muller A, Michel L, Basset-Seguin N, Modat G, Dubertret L, Bonne C. Characterization of specific leukotriene $\mathrm{C}_{4}$ binding sites on cultured human keratinocytes. $\mathrm{Br} J \mathrm{Der}$ matol 1988; 119 : 275-80. des phospho-inositides, est également impliqué dans la transduction du signal $\mathrm{LTB}_{4}[14]$. Dans les polynucléaires neutrophiles, l'activation de la protéine kinase $\mathrm{C}$ (PKC) par le DG induit la régulation négative des récepteurs du $\mathrm{LTB}_{4}[15]$, mais est aussi probablement responsable de certains effets de ce leucotriène.

$\mathbf{L T D}_{4} / \mathbf{E}_{4}$. Le mécanisme de couplage des signaux $\mathrm{LTD}_{4}$ présente une grande analogie avec celui du $\mathrm{LTB}_{4}[16-18]$. Dans différents muscles cibles, le $\mathrm{LTD}_{4}$ induit une accumulation rapide d'IP, et de calcium. L'hydrolyse du phosphatidylinositol (PI) précède la contraction musculaire ainsi que la libération de prostanoïdes, également impliqués dans la réponse biologique au leucotriène. Tous ces effets sont inhibés par le IAP.

Une bonne corrélation a été établie entre l'affinité d'analogues de synthèse agonistes du $\mathrm{LTD}_{4}$ et leur capacité à stimuler la synthèse d'IP, Enfin, des antagonistes, synthétisés par différents groupes, inhibent parallèlement la liaison du $\mathrm{LTD}_{4}$ à ses sites, l'hydrolyse du PI et la contraction musculaire.

\section{Effets liés à la formation de prostanoïdes}

Dans certains modèles expérimentaux, des prostanoïdes (métabolites de l'acide arachidonique par la voie de la cyclo-oxygénase) peuvent être impliqués comme médiateurs secondaires des effets induits par les leucotriènes. En particulier, la contraction du parenchyme pulmonaire est due partiellement à la synthèse de thromboxane $\mathrm{A}_{2}\left(\mathrm{TXA}_{2}\right)$ [19]. De même, nous avons montré que l'activation de la croissance de cellules endothéliales artérielles en culture provoquée par le $\mathrm{LTC}_{4}$ et le $\mathrm{LTD}_{4}$ était en partie liée à la formation de produits de la cyclo-oxygénase [20]. Le mécanisme par lequel les leucotriènes activent cette synthèse n'est certainement pas unique; toutefois, il a été démontré sur des cellules musculaires lisses en culture $\left(\mathrm{BC}_{3} \mathrm{H}_{1}\right)$ que la production de thromboxane et la contraction induites par le $\mathrm{LTC}_{4}$ et le $\mathrm{LTD}_{4}$ sont bloquées par le cycloheximide et l'actinomycine $\mathrm{D}[21]$. Ces résultats montrent que le méta- 
bolisme de l'acide arachidonique, provoqué par ces leucotriènes, pourrait nécessiter la synthèse d'une protéine activatrice de la phospholipase $\mathrm{A}_{2}$ [22].

\section{Autres mécanismes}

Les récepteurs des leucotriènes sont par ailleurs différemment couplés à l'adénylate cyclase. L'augmentation du taux intracellulaire de l'AMP cyclique par le $\mathrm{LTB}_{4}$ peut être interprétée comme un mécanisme secondaire de rétrocontrôle négatif [23]. Inversement, la diminution d'AMP cyclique, parfois observée sous l'effet du $\mathrm{LTD}_{4}$, pourrait concourir à l'activation cellulaire induite par ce peptidoleucotriène.

Il a été suggéré que le $\mathrm{LTB}_{4}$ ou ses produits d'oxydation pourraient agir aussi par simple effet ionophore calcique [24, 25]. Ceci rendrait compte des effets prolifératifs de ce leucotriène sur des cellules telles que les kératinocytes, où aucun site de liaison n'a pu être caractérisé [26].

\section{Conclusion}

Les mécanismes empruntés par les leucotriènes $\mathrm{LTB}_{4}$ et $\mathrm{LTD}_{4}$ pour transmettre leurs messages à leurs cellules cibles semblent communs à beaucoup d'autres molécules informatives (voir m/s $n^{\circ} 4$, vol. 1, p. 192 ; $n^{\circ} 5$, vol. 1, p. $255 ; n^{\circ} 3$, vol.3, p. $175 ; n^{\circ} 10$, vol. 3, p. 566) avec cependant des particularités, qui ne sont certainement pas toutes élucidées. Il reste surtout de nombreuses inconnues en ce qui concerne le rôle propre du $\mathrm{LTC}_{4}$ et son mécanisme d'action dont on ne sait seulement, aujourd'hui, que ce qu'il n'est pas! La découverte des leucotriènes, médiateurs de l'inflammation et de l'asthme, a suscité de nombreuses recherches pharmacologiques dans le but d'inhiber leur synthèse et, plus récemment, dans celui de bloquer spécifiquement leurs effets. La connaissance de leurs récepteurs respectifs a conduit divers groupes* à synthétiser et à sélectionner plusieurs antagonistes, dont l'évaluation clinique est en cours

* SKF, ICI, E. Lilly, MSD, ONO, Wyeth, Gresalem GS-CNRS leucotriènes.

$\mathrm{m} / \mathrm{s} n^{\circ} 1$ vol. 5 , janvier 89

\section{Summary}

Leukotrienes are arachidonic acid metabolites which have been identified as constituents of the Slow Reacting Substance of Anaphylaxis. At present they appear to be ubiquitous modulators of numerous cell functions. Their mechanisms of action are beginning to be elucidated. $\mathrm{LTB}_{4}$ and $\mathrm{LTD}_{4}$ interact with high affinity specific receptors on their respective target cells (e.g. LTB $_{4}$ : neutrophils, $\mathrm{LTD}_{4}$ : airway smooth muscles) and activate the phosphoinositide cycle. Inositol triphosphate and diacylglycerol are the main second messengers of these molecules. They also trigger the release of arachidonic acid from membrane phospholipids and act in part via cyclooxygenase products, e.g. thromboxane $A_{2}$. LTE $_{4}$ possesses the same target cells as $\mathrm{LTD}_{4}$ and seems to be active by binding to $\mathrm{LTD}_{4}$ receptors. In contrast, $\mathrm{LTC}_{4}$ has its own specific binding sites. They are distributed in the external side of the plasma membrane and also in intracellular membranes and mitochondrias. No clear transduction mechanism has yet been characterized for this leukotriene, which however induces specific effects.

\section{TIRÉS A PART}

C. Bonne. 